\title{
JANVIER 1976
}

58॰ ANNÉE - N` 685
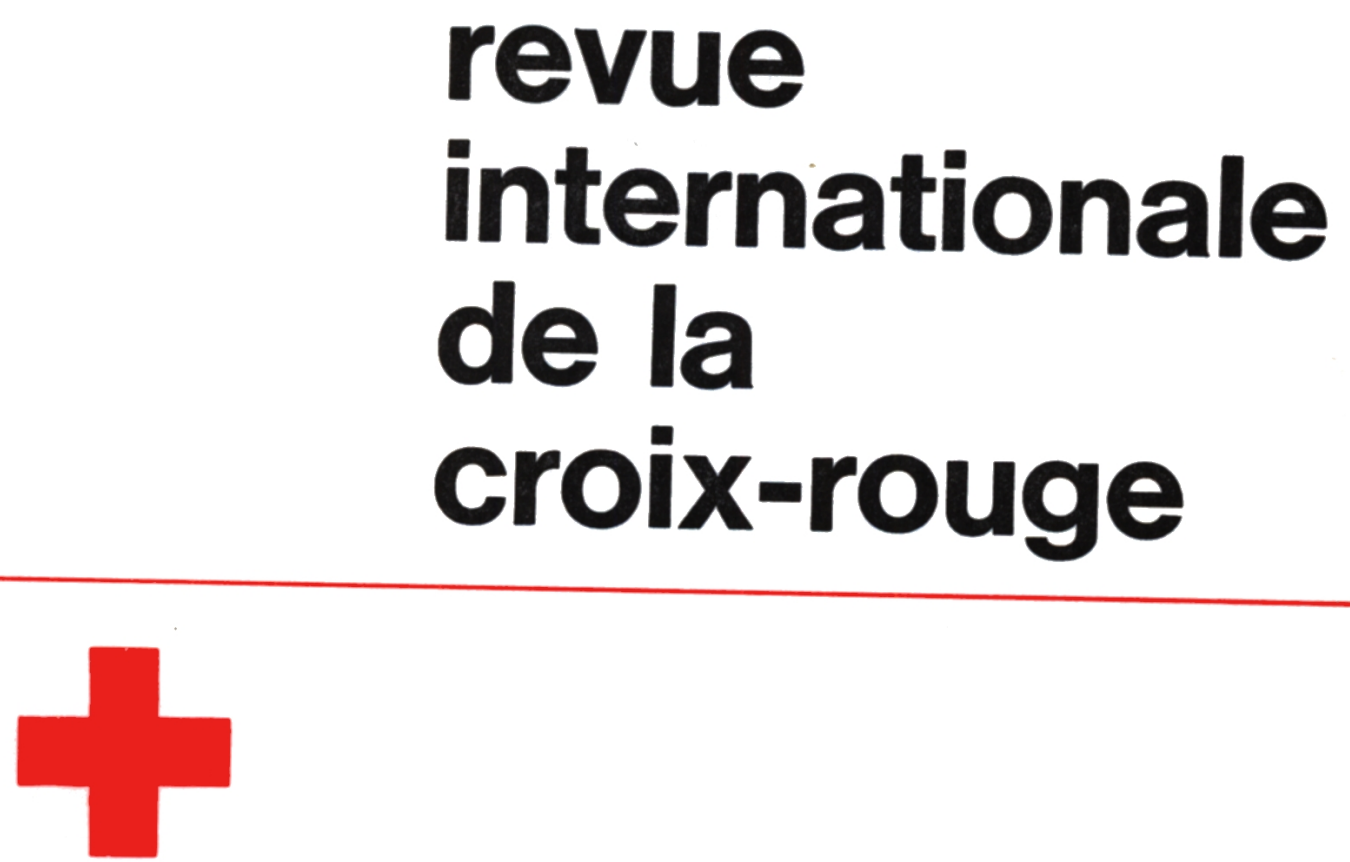

INTER ARMA CARITAS

GENÈVE

COMITÉ INTERNATIONAL DE LA CROIX-ROUGE FONDÉ EN 1863 


\section{COMITÉ INTERNATIONAL DE LA CROIX-ROUGE}

MM. ERIC MARTIN, docteur en médecine, professeur honoraire de l'Université, Genève, président (membre depuis 1973)

JEAN PICTET, docteur en droit, président de la Commission juridique, directeur de l'Institut Henry-Dunant, professeur associé à l'Université, Genève, vice-président (1967)

HARALD HUBER, docteur en droit, juge fédéral, vice-président (1969)

HANS BACHMANN, docteur en droit, directeur des finances de la ville, Winterthour (1958)

$M^{\text {me }}$ DENISE BINDSCHEDLER-ROBERT, docteur en droit, professeur à l'Institut universitaire de hautes études internationales, Genève, juge à la Cour européenne des droits de l'homme (1967)

MM. MARCEL A. NAVILLE, licencié ès lettres, président du CICR de 1969 à 1973 (1967)

JACQUES F. DE ROUGEMONT, docteur en médecine (1967)

ROGER GALLOPIN, docteur en droit, ancien directeur général du CICR (1967)

WALDEMAR JUCKER, docteur en droit, secrétaire de l'Union syndicale suisse (1967)

VICTOR H. UMBRICHT, docteur en droit, administrateur (1970)

PIERRE MICHELI, licencié en droit, ancien ambassadeur (1971)

GILBERT ETIENNE, professeur à l'Institut universitaire de hautes études internationales et à l'Institut d'études du développement, Genève (1973)

ULRICH MIDDENDORP, docteur en médecine, chef de la clinique chirurgicale de l'Hôpital cantonal, Winterthour (1973)

Mme MARION BOVÉE-ROTHENBACH, diplômée M.S.W. de l'Université de Michigan, maîtreassistant à l'Ecole des sciences sociales et politiques de l'Université, Lausanne (1973)

MM. HANS PETER TSCHUDI, docteur en droit, ancien conseiller fédéral (1973)

HENRY HUGUENIN, directeur de banque (1974)

GOTTFRIED DE SMIT, administrateur (1974)

JAKOB BURCKHARDT, docteur en droit, ministre plénipotentiaire, président du Conseil des Ecoles poly techniques fédérales (1975)

THOMAS FLEINER, docteur en droit, professeur à l'Université de Fribourg (1975)

ALEXANDRE HAY, avocat, directeur général de la Banque nationale suisse, président élu, (1975)

HERBERT LUTHY, docteur en philosophie, professeur d'histoire à l'Université de Bâle (1975)

Membres honoraires: M. JACQUES CHENEVIÈRE, vice-président d'honneur; $\mathrm{M}^{11 \mathrm{e}}$ LUCIE ODIER, vice-présidente d'honneur; MM. GUILLAUME BORDIER, PAUL CARRY, $M^{\text {me }}$ MARGUERITE GAUTIER-VAN BERCHEM, MM. ADOLPHE GRAEDEL, EDOUARD DE HALler, RODOlFo OLGIATI, MAX PETITPIERRE, PAUl RUEGger, DIETRICH SCHINDLER, FRÉDERIC SIORDET, ALFREDO VANNOTTI.

\section{CONSEIL EXÉCUTIF}

M. ROGER GALLOPIN, président

M. VICTOR H. UMBRICHT, vice-président

$M^{\text {me }}$ DENISE BINDSCHEDLER-ROBERT

M. GILBERT ETIENNE

$D^{\text {T ULRICH MIDDENDORP }}$

M. JEAN PICTET

M. GOTTFRIED DE SMIT

M. PIERRE MICHELI, membre suppleant 


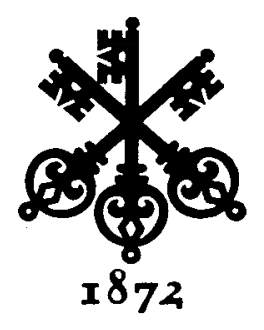

PRÉSENTE SUR LES CINQ CONTINENTS, LA GRANDE BANQUE AUX TROIS CLEFS EST À VOTRE DISPOSITION POUR EFFECTUER TOUTES VOS OPÉRATIONS FINANCIĖRES

\section{SOCIÉTÉ DE BANQUE SUISSE}

SCHWEIZERISCHER BANKVEREIN - SOCIETA DI BANCA SVIZZERA SWISS BANK CORPORATION

SIĖGE DE GENÈVE 2, RUE DE LA CONFÉdÉRATION TÉL. 224111

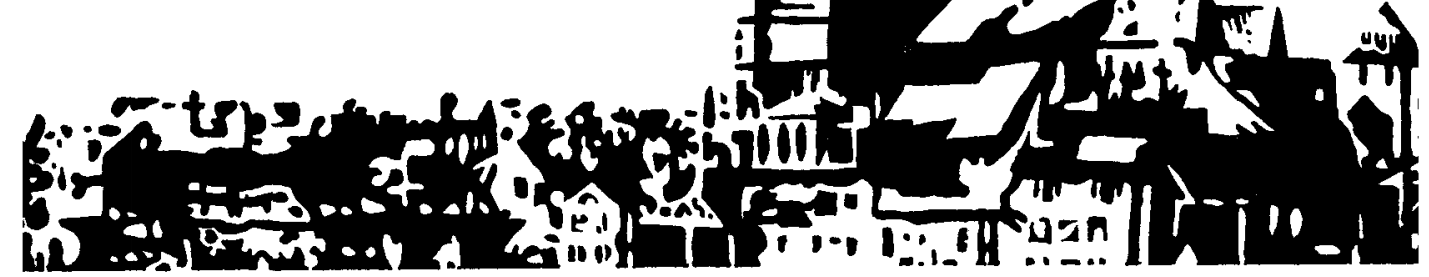




\title{
SOCIETE FIDUCIAIRE ROMANDE OFOR S.A.
}

\author{
Place Saint-Gervais 1, Genève - Tél. 317050
}

— Expertises, révisions et organisations comptables

$\downarrow$ Domiciliation et administration de sociétés

$\downarrow$ Assainissements et liquidations

\ Interventions et conseils en matière fiscale

\section{JOURIAL DE GEIEVE}

deux imprimeries deux adresses

rue François-Dussaud 20

1211 Genève 8 - Téléphone 431940

rue Général-Dufour 5-7

1211 Genève 11 - Tóléphone 250350

TYPO - OFFSET - ROTATIVE 


\section{REVUE INTERNATIONALE \\ DE LA CROIX-ROUGE}

JAN VIER 1976 - N685

COMITE INTERNATIONAL DE LA CROIX-ROUGE

DANS LE MONDE DE LA CROIX-ROUGE

FAITS ET DOCUMENTS
Paul Ruegger : Gustave Moynier

Activités extérieures:

Afrique - Amérique latine - Asie - Moyen-

Orient - Europe . . . . . . . . . . . .

A Genève:

Participations aux Conventions de Genève . . . 25

Deux publications en langue arabe . . . . 26

Diffusion des Conventions de Genève: Ghana-

Nigéria-Pologne . . . . . . . . . . . 30

Institut Henry-Dunant . . . . . . . . . . . . 34

L'aide de la Croix-Rouge internationale au Vietnam . . . . . . . . . . . . . . . 37

La Croix-Rouge internationale rapatrie des étrangers . . . . . . . . . . . . . . . 38

Journée mondiale de la Croix-Rouge . . . . . 40

République de Corée . . . . . . . . . . . . 41

Libye . . . . . . . . . . . . . . . . . . . 42

Année internationale de la femme . . . . . . . 45

Résolutions des Nations-Unies sur le développement du droit international humanitaire . . 50 


\section{INTERNATIONAL \\ REVIEW \\ OF THE RED CROSS}

REVISTA INTERNACIONAL DE LA CRUZ ROJA

\section{EXTRAITS}

DE LA REVUE

EN LANGUE ALLEMANDE

\section{REVUE INTERNATIONALE DE LA CROIX-ROUGE}

Une édition en langue anglaise et une en langue espagnole paraissent chaque mois. Elles sont en principe identiques à l'édition française, et peuvent être obtenues aux mêmes conditions.

Ein neues Inhaltsverzeichnis der « Revue Internationale de la Croix-Rouge » für die Jahre 1962-1974 — Neue Beitritte zu den Genfer Abkommen - Henry-Dunant Institut - Niederlande - Die Hilfe des internationalen Roten Kreuzes in Vietnam.

Elle est publiée chaque mois par le Comité international de la Croix-Rouge.

17, avenue de la Paix, 1211 Genève (Suisse) - Compte de chèques postaux $12-1767$.

Abonnement un an: Fr. 30,-; le numéro: Fr. 3,-

RÉDACTION: J.-G. LOSSIER

Seuls les textes signés par le Comité international de la Croix-Rouge engagent la responsabilité de celui-ci. 